\title{
British Thoracic Society guidelines for the management of spontaneous pneumothorax: do we comply with them and do they work?
}

\author{
Thomas Soulsby
}

\begin{abstract}
Objectives-To determine how British Thoracic Society (BTS) guidelines for the management of spontaneous pneumothorax have been implemented over a four year period in a large district general hospital with a specialised respiratory unit. To assess the validity of the guidelines in the management of spontaneous pneumothorax.
\end{abstract}

Methods-A retrospective case notes study over a four year period for all patients with spontaneous pneumothoraces attending the accident and emergency (A\&E) department of a large district general hospital.

Results-115 episodes of spontaneous pneumothorax were identified. Overall $20.8 \%$ of episodes were treated correctly by BTS guidelines. Decisions made by A\&E staff were significantly more likely to be correct than decisions made by on call medical teams $(39.4 \%$ v $13.4 \%$, $\mathbf{p}=0.002$ ). There was a non-significant trend towards better compliance with BTS guidelines over the four year period. The management decision was made by A\&E staff only in $28.7 \%$ of episodes. Patients without chronic lung disease could be safely observed as outpatients. Forty three pneumothoraces were aspirated: $23(58 \%)$ successfully; eight $(18.6 \%)$ with little or no improvement; and 12 $(27.9 \%)$ initially successfully but developing increased size of pneumothorax within a period of 72 hours. Three patients had repeat aspirations, two successfully. Age over 50, chronic lung disease, and more than 2.5 litres of air aspirated were significantly associated with failure of aspiration $(p<0.01)$. There was a $28.6 \%$ failure rate of aspiration for patients with

Accepted for publication 28 May 1998 moderate pneumothoraces without chronic lung disease.

Conclusions-Patients over the age of 50 should be treated the same as patients with chronic lung disease. A second aspiration should be attempted if the first aspiration is initially successful with subsequent recurrence of pneumothorax. Further research is required to clarify if patients with moderate pneumothoraces under the age of 50 and no chronic lung disease should be aspirated or observed. (F Accid Emerg Med 1998;15:317-321)

Keywords: pneumothorax; aspiration

Before the introduction of British Thoracic Society (BTS) guidelines for the management of spontaneous pneumothoraces in 1993,' intercostal drainage was the standard treatment for moderate or large pneumothoraces. This was despite eight papers (all series of patients, none randomised) showing success rates between $53 \%$ and $84 \%$ for needle aspiration. ${ }^{2-10}$ Features found in several of these papers to predict success of aspiration were age under 50 , absence of chronic lung disease, size of pneumothorax, and volume of air aspirated. ${ }^{247910}$

The BTS guidelines (devised by the Standards of Care Committee of the BTS) were intended to assist in the immediate management of patients presenting to "casualty departments". A flow diagram was produced to cover immediate management (fig 1). The guidelines were intended to be used on a national basis and were based on a randomised trial of 73 patients and the opinions of over 150 British respiratory physicians and thoracic surgeons. The trial randomised patients between aspiration and intercostal drainage. ${ }^{11}$ Patients were excluded who had signs of tension or with previous lung disease (other than previous pneumothorax). Pneumothoraces were graded into small rim, partial collapse, or complete collapse; $80 \%$ of the 35 patients were aspirated successfully (although five patients required two aspirations). The amount of air aspirated was highly significantly different in successful aspirations (1.59 litres) compared with unsuccessful (2.52 litres). There was no significant association between successful aspiration and the size of pneumothorax on initial radiography.

The authors' comment was that aspiration should be the initial treatment of choice for patients with normal lungs who present with a

Figure 1 BTS guidelines. 


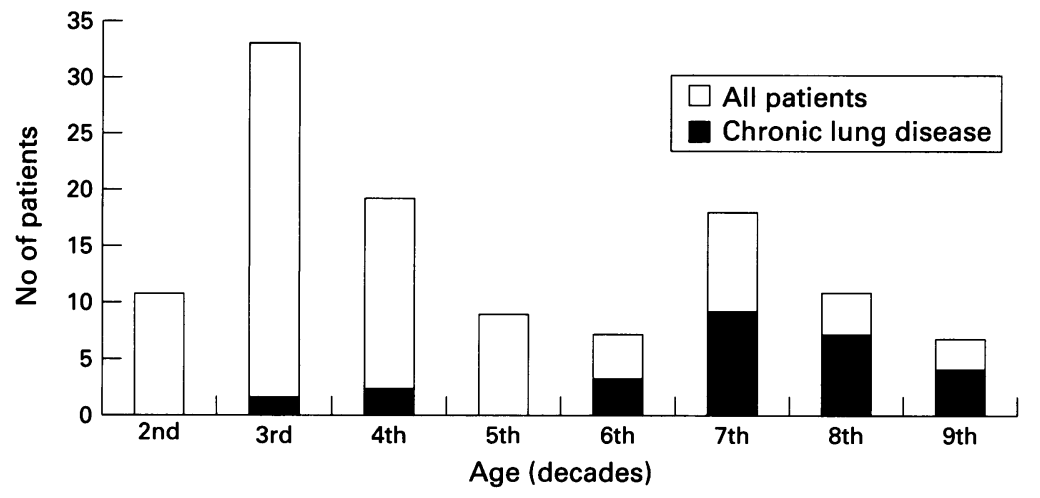

Figure 2 Age and chronic lung disease distribution.

Table 1 Initial treatment by size of pneumothorax

\begin{tabular}{llllll}
\hline \multirow{2}{*}{ Treatment group } & \multicolumn{2}{l}{ Size of pneumothorax } & & \\
\cline { 2 - 5 } & Small & Moderate & Complete & Not known & Total \\
\hline Observation & 12 & 9 & 0 & & 21 \\
Aspiration & 6 & 29 & 7 & 1 & 43 \\
Drain & 2 & 16 & 30 & 3 & 51 \\
\hline
\end{tabular}

spontaneous pneumothorax, irrespective of its size. However the guidelines (published over a year previously) included patients with preexisting lung disease and recommend aspiration (in patients with no previous lung disease) only with a complete pneumothorax unless "significant dyspnoea" is present. Significant dyspnoea was defined as "an obvious deterioration in usual exercise tolerance". A maximum figure for aspiration of $2500 \mathrm{ml}$ of air was given and a repeat aspiration was only recommended if it was thought the cannula had been kinked or withdrawn inadvertently from the pleural cavity. Patients without chronic lung disease could be sent home after aspiration.

There has been one further prospective randomised trial published comparing aspiration with intercostal drainage. ${ }^{12}$ This however compared delayed needle aspiration at three days in the randomised part of the trial. A subsequent series of patients were then treated by immediate needle aspiration. Since the guidelines were published in 1993 there have been no reports published showing whether they are being used in clinical practice. The aim of this study was to audit compliance with BTS guidelines and to assess the validity of the guidelines.

\section{Methods}

A retrospective survey was carried out of case notes for all patients seen with a diagnosis of pneumothorax over a four year period. Case notes were identified by International Classification of Diseases diagnosis codes from the accident and emergency (A\&E) department computer system and from discharge diagnosis codes from the wards (including the observation ward).

Case notes were analysed with a standardised proforma. Pneumothoraces were graded by size into small, moderate, or complete (according to BTS guidelines). Patients were put into one of three treatment groups: observation, aspiration, or chest drain as the initial treatment. Inpatient observation was assessed as incorrect by BTS guidelines if there was no diagnosis of chronic lung disease. If the patient was observed as an inpatient on a medical ward (usually the chest unit) it was counted as a "medical decision". If the patient was admitted to the observation ward or reviewed in $A \& E$ it counted as an $\mathrm{A} \& \mathrm{E}$ decision.

All patients aspirated who had chronic lung disease were deemed as correct by BTS guidelines. For patients without chronic lung disease, if they had a documented decrease in exercise tolerance or were dyspnoeic on examination, the decision to aspirate was deemed as correct. All patients who had a chest drain as an initial manoeuvre were deemed as incorrect.

Results were analysed by the Aintree Hospitals Trust statistician using the SPSS software package. The $\chi^{2}$ and Pearson's tests were used; $p$ values of less than 0.05 were considered significant.

\section{Results}

A total of 209 records were identified. Of these $176(84 \%)$ case notes were available; 61 were excluded due to trauma, tension, or were iatrogenic. There were 115 episodes of spontaneous pneumothorax in 97 patients.

The sex distribution, age, and presence of chronic lung disease were similar to previous reported series. Age and chronic lung disease distribution are shown in fig 2. Age distribution had two peaks: in the second and seventh decades. Over the age of 50 chronic lung disease predominated. Forty (34.8\%) episodes involved patients with previous pneumothoraces.

Symptoms noted were dyspnoea in 89 $(77.4 \%)$ patients and chest pain in $82(71.3 \%)$ patients. There were no significant differences in age, chronic lung disease, or chest pain within the three treatment groups. There was no significant difference in the presence of dyspnoea between the aspiration and chest drain groups but significantly less in the observation group $(p<0.01)$. The size of pneumothorax for the three treatment groups are shown in table 1. Patients presenting with a complete pneumothorax were significantly more likely to have a chest drain inserted than to be aspirated $(\mathrm{p}<0.01)$.

Overall $24(20.8 \%)$ episodes were treated correctly by BTS guidelines. Table 2 shows

Table 2 Initial treatment correct by BTS guidelines; values are number (\%)

\begin{tabular}{llllll}
\hline 12 months starting: & No of episodes & Observation & Aspiration & Drain & $\begin{array}{l}\text { Correct by BTS } \\
\text { guidelines }\end{array}$ \\
\hline August 93 & 19 & $4(21 . .0)$ & $5(26.3)$ & $10(52.7)$ & $1(5.3)$ \\
August 94 & 38 & $11(29.0)$ & $14(37.0)$ & $13(34.0)$ & $9(23.7)$ \\
August 95 & 47 & $5(10.6)$ & $20(42.0)$ & $22(46.8)$ & $10(21.3)$ \\
August 96 & 11 & $1(9.1)$ & $4(36.4)$ & $6(54.5)$ & $4(36.4)$ \\
\hline
\end{tabular}


Table 3 Decision by specialty, correct by BTS guidelines and by initial treatment

\begin{tabular}{llllll}
\hline Initial treatment & Total & $\begin{array}{l}\text { AEE } \\
\text { decision }\end{array}$ & $\begin{array}{l}\text { Correct by } \\
\text { BTS }\end{array}$ & $\begin{array}{l}\text { Medical } \\
\text { team } \\
\text { decision }\end{array}$ & $\begin{array}{l}\text { Correct by } \\
\text { BTS }\end{array}$ \\
\hline Observation & 21 & 3 & 3 & 18 & 2 \\
Aspiration & 43 & 17 & 10 & 26 & 9 \\
Drain & 51 & 13 & 0 & 38 & 0 \\
Total (\%) & 115 & 33 & $13(39.4)$ & 82 & $11(13.4)$ \\
\hline
\end{tabular}

Table 4 Success of aspiration

\begin{tabular}{llll}
\hline Size of pneumothorax & $\begin{array}{l}\text { Successful } \\
\text { (no chest drain) }\end{array}$ & Immediate failure & Late failure \\
\hline Small & 5 & 1 & 0 \\
Moderate & 18 & 5 & 8 \\
Complete & 2 & 2 & 4 \\
All sizes (\%) & $25(58.0)$ & $8(18.6)$ & $12(27.9)$ \\
\hline
\end{tabular}

Table 5 Success of aspiration by age, chronic lung disease, and volume of air aspirated; values are $\%$

\begin{tabular}{llllll}
\hline Result of aspiration & Age $<50$ & Age $>50$ & $\begin{array}{l}\text { Chronic } \\
\text { lung disease }\end{array}$ & $\begin{array}{l}>2.5 \text { litres } \\
\text { air aspirated }\end{array}$ & $\begin{array}{l}<2.5 \text { litres } \\
\text { air aspirated }\end{array}$ \\
\hline Successful & 70.4 & 31.3 & 27.3 & 0 & 71.4 \\
Immediate failure & 3.7 & 50.0 & 54.5 & 50.0 & 14.3 \\
Late failure & 25.9 & 18.8 & 18.2 & 50.0 & 14.3 \\
\hline
\end{tabular}

Table 6 Volume of air aspirated in moderate

pneumothoraces (without chronic lung disease) compared to success of aspiration; values are number (\%)

\begin{tabular}{lll}
\hline $\begin{array}{l}\text { Volume aspirated } \\
\text { litres) }\end{array}$ & Successful & Unsuccessful \\
\hline$<1.0$ & $4(80.0)$ & $1(20.0)$ \\
$1.0-2.0$ & $8(88.9)$ & $1(11.1)$ \\
$>2.0$ & & $3(100.0)$ \\
\hline
\end{tabular}

how this varied in 12 month periods since introduction in August 1993 up to 1997. There was a non-significant trend towards better compliance with BTS guidelines over the four year period. It should be noted however that the percentage being treated initially with a chest drain remained constant. The improved compliance was due to fewer patients with small and moderate pneumothoraces being aspirated.

Of the 115 treatment decisions, 33 (28.7\%) were made by $A \& E$ and $82(71.3 \%)$ were made by the on call medical team. Table 3 shows which of these decisions were correct by BTS guidelines. Overall $A \& E$ decisions were significantly more likely to be correct compared with those by the on call medical team $(\mathrm{p}=0.002)$.

The observation group had one patient who required intervention subsequent to admission. This patient had chronic lung disease and subsequently received aspiration and then intercostal drainage. The 16 patients without chronic lung disease in the observation group had no interventions and could have been safely sent home.

Forty three patients were aspirated; the results are shown in table 4 . Immediate failure is where there was small or no improvement in the pneumothorax. The 12 patients in the late failure group had an initially successful aspiration and then an increased size of pneumothorax (all within 72 hours). Three had a repeat aspiration (two were successful) and 10 received intercostal drainage. The one small pneumothorax unsuccessfully aspirated was in a patient with chronic obstructive pulmonary disease who subsequently required intercostal drainage for 26 days on suction and pleurodesis. There was a non-significant trend towards failure in patients with complete pneumothoraces (success rate $28.6 \%$ ).

Age over $50(p=0.001)$, chronic lung disease $(\mathrm{p}=0.006)$, and more than 2.5 litres of air aspirated $(p=0.002)$ were all significantly associated with failure of aspiration (table 5). Excluding patients with chronic lung disease from the group aged over 50 left five patients. The success rate was $40 \%$ for aspiration which is similar to the group of patients with chronic lung disease $(27.3 \%)$. All patients who had more that 2.5 litres of air aspirated ended up with an intercostal drain.

There were 21 patients with a moderate pneumothorax without chronic lung disease who were aspirated. This is the group of patients who should only be aspirated if complaining of significant dyspnoea according to BTS guidelines: $17(81 \%)$ complained of dyspnoea, but only one was noted to have decreased exercise tolerance. Fifteen patients $(71.4 \%)$ were successfully aspirated. In the six that failed, one failed immediately and the other five developed recurrent pneumothoraces over a period of 72 hours. The amounts aspirated for the whole group are shown in table 6. All patients with over 21 aspirated received intercostal drainage.

Overall there was a significant difference between the three treatment groups for need for surgery during that admission, complications, and eventual pleurectomy $(p<0.001$; table 7). If just those patients in the aspiration group who had intercostal drainage are compared with those in the group that had drains as initial treatment there was no difference in complications, unsuccessful intercostal drainage, and length of insertion of drain. There were no complications described for aspiration. Recurrence was not significantly different in the three treatment groups. The length of stay was significantly longer for the intercostal drainage group $(p<0.001)$.

\section{Discussion}

The BTS guidelines have been an important step forward in the management of spontaneous pneumothorax. The compliance with BTS guidelines was poor $(20.8 \%)$. However patients with moderate pneumothoraces and no chronic lung disease may have had "significant

Table 7 Complications, surgery, and length of stay by treatment group

\begin{tabular}{lllll}
\hline & Complications (\%) & Unsuccessful drain (\%) & $\begin{array}{l}\text { Eventual pleurectomy } \\
(\%)\end{array}$ & $\begin{array}{l}\text { Length of stay (median in } \\
\text { days) }\end{array}$ \\
\hline Aspiration & 7.0 & 4.6 & 11.6 & 3.9 \\
Drain & 18.8 & 19.6 & 25.5 & 8.0 \\
Observation & 0 & 0 & 9.5 & 2.0 \\
\hline
\end{tabular}


dyspnoea" despite it not being recorded in the notes. If patients without chronic lung disease who had moderate pneumothoraces aspirated were interpreted as all being treated correctly then the compliance with BTS guidelines improved to $36.5 \%$. If patients were admitted after aspiration the treatment decision was still assessed as correct. This is because it is local policy within the hospital to admit patients after aspiration (although BTS guidelines recommend outpatient follow up for patients without chronic lung disease).

The group treated with chest drains initially was managed incorrectly by BTS guidelines. These patients were exposed to a painful procedure, potential morbidity, and a longer stay in hospital. This group comprised 51 patients. With a success rate of $60 \%$ for aspiration it can be estimated that at least 29 patients may not have required chest drains. The use of chest drains as the initial procedure may be explained by several factors. Historically, insertion of a chest drain was the usual treatment for moderate or large pneumothoraces. A retrospective case note audit of patients treated in 1991 in Scotland showed aspiration only being used in three patients out of 37, compared with 21 patients receiving intercostal drainage. ${ }^{13}$ Doctors may be unaware of the BTS guidelines. The preponderance of patients with a complete pneumothorax in this group suggests that although doctors are aware of the use of needle aspiration, they do not think it will be successful for complete pneumothoraces.

The observation group represents a tendency for caution. Only five of the 21 patients in this group required admission by BTS guidelines. While this is costly for use of beds at least no harm is done to the patient.

Of the 115 patients, $82(71.3 \%)$ had management decisions made by the on call medical team. It is not clear why this figure is so high or whether similar figures would be found in other $A \& E$ departments. It may reflect the fact that when the $A \& E$ department is busy there is pressure on the $A \& E$ staff to leave treatment to a specialist team and to continue seeing more $A \& E$ patients. It could also be due to junior $A \& E$ staff not feeling capable of dealing with insertion of chest drains.

The difference in compliance with BTS guidelines between $A \& E$ staff and the on call medical teams may be partially explained by the greater number of patients admitted for observation to the chest unit compared with the observation ward (incorrectly by BTS guidelines). There was no significant difference for chest drain insertion for A\&E staff compared with the medical teams (39\% $v 46 \%$ ). However A\&E staff were more likely to use aspiration than the medical teams $(51 \% v$ $31 \%)$. This was true for moderate and complete pneumothoraces.

The smaller number of patients seen with pneumothorax from August 1996 seems to represent a genuine fall in numbers. There was no significant difference in the distribution of the size of pneumothorax for each 12 month period. It is possible that some patients were discharged from the $A \& E$ department without intervention and have been missed as the $A \& E$ department computer system doesn't cover the last $\mathbf{1 2}$ months. It is unlikely that patients were aspirated and discharged (due to local policy). For those patients admitted over the last 12 month period, $54.5 \%$ still received a chest drain as the initial treatment.

This study confirms that when aspiration is used it is safe and can be effective for all sizes of pneumothorax, although there is a lower success rate for complete pneumothoraces (non-significant but only eight patients).

All patients admitted for observation without chronic lung disease did not receive treatment and could have been safely sent home. The recommendation that patients without chronic lung disease can be sent home after aspiration is safe in that no patients developed tension pneumothoraces. However $25 \%$ of patients in this group developed increased size of pneumothorax within 72 hours which required further treatment (either repeat aspiration or intercostal drainage).

The presence of dyspnoea as a symptom did not differ significantly for small, moderate, or large pneumothoraces. Patients with chronic lung disease were frequently noted to be dyspnoeic on examination and have abnormal arterial blood gases. This was not the case in "simple pneumothorax" and only one patient was documented as having reduced exercise tolerance. This may be partly due to the study being retrospective. It also reflects the difficulty of defining "significant dyspnoea" in patients who do not appear dyspnoeic on examination but have pleuritic chest pain. Many of these patients attend hospital shortly after onset of symptoms and there may be no definite decrease in exercise tolerance. Very few patients had a record of oxygen saturation and no comment can be made as to whether this may be used to indicate a need for intervention.

The success rate for aspiration is lower in the presence of chronic lung disease and increasing age. The incidence of pneumothorax has two peaks: the third and seventh decades. Patients over the age of 50 had similar results to those with chronic lung disease. This may reflect undiagnosed lung disease. The recommendation for use of aspiration in moderate pneumothoraces for patients with chronic lung disease is based on the principles that these patients tolerate pneumothoraces less well and are less likely to have a successful aspiration. ${ }^{1} \mathrm{~A}$ similar argument can be made for all patients over 50.

Estimating the size of pneumothorax is known to be imprecise, ${ }^{14}$ hence the simplification into three sizes. This means that a moderate pneumothorax may contain a large volume of air ( $70 \%$ over 1 litre). BTS guidelines only recommend aspiration for moderate pneumothoraces in patients without chronic lung disease when there is the presence of significant dyspnoea. Most of these patients complain of some dyspnoea. Considering the safety of the procedure (this group should be at low risk of adhesions) it may be that all these patients should be aspirated. This would mean fewer 
hospital visits (due to quicker resolution of pneumothorax). It is not clear from previous studies whether this group of patients in this series who had drain insertion due to failure of aspiration would have had resolution of the pneumothorax if no aspiration had been attempted and with sufficient time for observation as an outpatient. Stradling and Poole reported a series of 82 patients with "simple pneumothorax" observed for six weeks. ${ }^{2}$ Fourteen patients required intervention giving a success rate of $83 \%$ for observation (compared with a success rate of $71 \%$ for aspiration in this series for moderate pneumothoraces without chronic lung disease). However the 82 patients had been referred to a chest clinic (mostly via general practitioners) and the size of pneumothorax was not reported in the paper. It is possible that there was a smaller proportion of complete pneumothoraces thus giving a better success rate.

A second aspiration is only recommended by the guidelines if there has been a problem with the technique. Patients who have had 2.5 litres of air aspirated with only slight or no resolution of the pneumothorax (that is, immediate failure) clearly have a large air leak and require intercostal drainage. However for those patients who have a delayed failure (increased size of pneumothorax within the next 72 hours) a repeat aspiration may be successful. Only three patients in this study had a repeat aspiration but previous studies have reported similar findings. All patients received chest drains who had 2.5 litres or more aspirated. The role of a second aspiration in this group who have 2.5 litres aspirated with resolution of the pneumothorax initially is not clear.

The lower rate of subsequent pleurectomy in the aspiration group compared with the chest drain group is in keeping with the BTS findings. ${ }^{11}$ There was no significant difference in recurrence. However there was a significantly higher proportion of large pneumothoraces in the chest drain group decreasing the validity of these comparisons. The use of a trochar is recommended in the BTS guidelines, although not by the advanced trauma life support course, nor commonly used in $A \& E$ practice. As a potential cause of morbidity its use should be removed from the guidelines.

\section{Conclusions}

Compliance with BTS guidelines is poor and is not significantly improving. Patients are receiving intercostal drainage unnecessarily. Compliance with guidelines may be improved if responsibility for initial management is taken by $A \& E$ staff; the guidelines are given a high priority within departmental teaching programmes; and implementation is audited.

As a result of this study (and previous published data) the BTS guidelines should be modified: to treat patients over the age of 50 the same as patients with chronic lung disease; to aspirate a second time if under 2.5 litres is aspirated initially with resolution of pneumothorax; and to remove advice to use a trochar for insertion of chest drains.

Further research is needed to investigate three areas: if oxygen saturation monitoring could be used as an indication for aspiration rather than "significant dyspnoea"; if patients with moderate pneumothoraces under the age of 50 and no chronic lung disease should be aspirated or observed; and the role of a second aspiration when over 2.5 litres has been aspirated with resolution of pneumothorax.

1 Miller AC, Harvey JE. Guidelines for the management of spontaneous pneumothorax. BMJ 1993;307:114-6.

2 Stradling P, Poole G. Conservative management of spontaneous pneumothorax. Thorax 1966;21:145-9.

3 Raja OJ, Lalor AJ. Simple aspiration of spontaneous pneumothorax. Br J Dis Chest 1981;81:693-4.

4 Bevelaqua FA, Aranda C. Management of spontaneous pneumothorax with a small lumen catheter. Chest 1982;75:207-8.

5 Spencer Jones J. A place for aspiration in the treatment of spontaneous pneumothorax. Thorax 1985;40:66-7.

6 Delius RE, Obeid FN, Horst HM, et al. Catheter aspiration for simple pneumothorax. Arch Surg 1989;124:833-6.

7 Archer GJ, Hamilton AAD, Upadhyay R, et al. Results of simple aspiration of pneumothoraces. Br J Dis Chest 1985; 79:177-82.

8 Vallee P. Sequential treatment of a simple pneumothorax. Ann Emerg Med 1988;17:936-42.

9 Markos J. Pneumothorax; treatment by small lumen catheter. Aust N Z J Med 1990;20:775-81.

$10 \mathrm{Ng} \mathrm{AW}$. Simple aspiration of pneumothorax. Singapore Med J 1994;35:50-2.

11 Harvey J, Prescott RJ. Simple aspiration versus intercostal tube drainage for spontaneous pneumothorax in normal lungs. BMJ 1994;309:1338-9.

12 Andrivet $\mathrm{P}$, Djedainik, Teboul $\mathrm{JL}$, et al. Spontaneous pneumothorax; comparison of thoracic drainage versus immediate or delayed aspiration. Chest 1995;108:335-9.

13 Selby CD, Sudflow MF. Deficiencies of management of spontaneous pneumothoraces. Scott Med J 1994;39:75-7. 14 Engdahl O, Toft T, Boe J. Chest radiograph - a poor method for determining the size of a pneumothorax. Chest 1993;103:26-9. 